\title{
Sum rules for baryon decuplet magnetic moments
}

\author{
Soon-Tae Hong* \\ W. K. Kellogg Radiation Laboratory, California Institute of Technology, Pasadena, California 91125, USA \\ and Department of Science Education and Research Institute for Basic Sciences, Ewha Womans University, Seoul 120-750, Korea
} (Received 12 April 2007; published 29 November 2007)

\begin{abstract}
In chiral models with SU(3) group structure, baryon decuplet, and octet magnetic moments are evaluated by constructing their sum rules to yield theoretical predictions. In these sum rules we exploit six experimentally known baryon magnetic moments. Sum rules for flavor components and strange form factors of the octet and decuplet magnetic moments and decuplet-to-octet transition magnetic moments are also investigated.
\end{abstract}

\section{INTRODUCTION}

The internal structure of the nucleon is still a subject of great interest to both experimentalists and theorists. In 1933, Frisch and Stern [1] performed the first measurement of the magnetic moment of the proton and obtained the earliest experimental evidence for the internal structure of the nucleon. However, it was not until 40 years later that the quark structure of the nucleon was directly observed in deep inelastic electron scattering experiments and we still lack a quantitative theoretical understanding of these properties including the magnetic moments.

Since Coleman and Glashow [2] predicted the magnetic moments of the baryon octet about 40 years ago, there has been a lot of progress in both the theoretical paradigm and experimental verification for the baryon magnetic moments. The measurements of the baryon decuplet magnetic moments were reported for $\mu_{\Delta^{++}}$[3] and $\mu_{\Omega^{-}}$[4] to yield a new avenue for understanding the hadron structure. The magnetic moments of baryon decuplet have been theoretically investigated in several models such as the quenched lattice gauge theory [5], the quark models [6], the chiral bag model [7], the chiral perturbation theory [8], the QCD sum rules [9], the chiral quark model [10], and the chiral quark soliton model [11]. Moreover, by including the effect of decuplet intermediate states of spin-3/2 baryons explicitly, the heavy baryon chiral expansion of baryon octet magnetic moments [12] and charge radii [13] were investigated. The decuplet-to-octet transition magnetic moments have been also analyzed in the $1 / N_{c}$ expansion of QCD [14-16] and in the chiral quark soliton model [17].

Quite recently, the SAMPLE Collaboration [18] reported the experimental data of the proton strange form factor through parity violating electron scattering [19]. To be more precise, they measured the neutral weak form

*soonhong@ewha.ac.kr factors at a small momentum transfer $Q_{S}^{2}=$ $0.1(\mathrm{GeV} / \mathrm{c})^{2}$ to yield the proton strange magnetic form factor in units of Bohr nuclear magnetons (n.m.) $G_{M}^{s}=$ $+0.37 \pm 0.20 \pm 0.26 \pm 0.07$ n.m. [18]. The HAPPEX Collaboration later reported $G_{M}^{s}=+0.18 \pm 0.27$ n.m. [20]. Moreover, McKeown [21] has shown that the strange form factor of proton should be positive by using the conjecture that the up-quark effects are generally dominant in the flavor dependence of the nucleon properties. The chiral bag model [22] predicted first the positive value for the proton strange form factor [23].

In this paper, we will exploit the chiral bag model to predict baryon decuplet and octet magnetic moments and their strange form factors. This model calculation can share those of other skyrmion extended models with SU(3) group structure if sum rules are properly used. More specifically, in the chiral models, we will investigate the magnetic moments of baryon decuplet and octet, together with the decuplet-to-octet transition magnetic moments, in terms of their sum rules. We will also study the sum rules for the flavor components and strange form factors of the baryon magnetic moments. In Sec. II, in the adjoint representation we construct the sum rules of the baryon decuplet and octet magnetic moments and the transition magnetic moments in the SU(3) chiral models, and in Sec. III the sum rules for their flavor components and strange form factors in a model independent way at least in the category of the chiral models with the $\mathrm{SU}(3)$ flavor group. In the Appendix, we list the $u$ - and $d$-flavor components of the baryon magnetic moments and transition magnetic moments and their sum rules.

\section{MAGNETIC MOMENTS OF BARYON DECUPLET}

We start with the chiral bag model with the broken $U$-spin symmetry whose Lagrangian is of the form 


$$
\begin{aligned}
\mathcal{L} & =\mathcal{L}_{\mathrm{CS}}+\mathcal{L}_{\mathrm{CSB}}+\mathcal{L}_{\mathrm{FSB}}, \\
\mathcal{L}_{\mathrm{CS}} & =\bar{\psi} i \gamma^{\mu} \partial_{\mu} \psi \Theta_{B}-\frac{1}{2} \bar{\psi} U_{5} \psi \Delta_{B}+\left(-\frac{1}{4} f_{\pi}^{2} \operatorname{tr}\left(l_{\mu} l^{\mu}\right)+\frac{1}{32 e^{2}}\left[l_{\mu}, l_{\nu}\right]^{2}+\mathcal{L}_{\mathrm{WZW}}\right) \bar{\Theta}_{B}, \\
\mathcal{L}_{\mathrm{CSB}} & =-\bar{\psi} M \psi \Theta_{B}+\frac{1}{4} f_{\pi}^{2} m_{\pi}^{2} \operatorname{tr}\left(U+U^{\dagger}-2\right) \bar{\Theta}_{B}, \\
\mathcal{L}_{\mathrm{FSB}} & =\frac{1}{6} f_{\pi}^{2}\left(\chi^{2} m_{K}^{2}-m_{\pi}^{2}\right) \operatorname{tr}\left(\left(1-\sqrt{3} \lambda_{8}\right)\left(U+U^{\dagger}-2\right)\right) \bar{\Theta}_{B}-\frac{1}{12} f_{\pi}^{2}\left(\chi^{2}-1\right) \operatorname{tr}\left(\left(1-\sqrt{3} \lambda_{8}\right)\left(U l_{\mu} l^{\mu}+l_{\mu} l^{\mu} U^{\dagger}\right)\right) \bar{\Theta}_{B},
\end{aligned}
$$

where the quark field $\psi$ has SU(3) flavor degrees of freedom and the chiral field $U=e^{i \lambda_{a} \pi_{a} / f_{\pi}} \in \mathrm{SU}(3)$ is described by the pseudoscalar meson fields $\pi_{a}$ $(a=1, \ldots, 8)$ and Gell-Mann matrices $\lambda_{a}$ with $\lambda_{a} \lambda_{b}=$ $\frac{2}{3} \delta_{a b}+\left(i f_{a b c}+d_{a b c}\right) \lambda_{c}$, and $\Theta_{B}\left(=1-\bar{\Theta}_{B}\right)$ is the bag theta function (one inside the bag and zero outside the bag). In the limit of vanishing bag radius, the chiral bag model is reduced to the Skyrmion model. Here $l_{\mu}=$

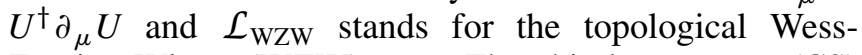
Zumino-Witten (WZW) term. The chiral symmetry (CS) is broken by the quark masses $M=\operatorname{diag}\left(m_{u}, m_{d}, m_{s}\right)$ and pion mass $m_{\pi}$ in $\mathcal{L}_{\mathrm{CSB}}$. Furthermore the $\mathrm{SU}(3)$ flavor symmetry breaking (FSB) with $m_{K} / m_{\pi} \neq 1$ and $\chi=$ $f_{K} / f_{\pi} \neq 1$ is included in $\mathcal{L}_{\mathrm{FSB}}$. Even though the mass terms in $\mathcal{L}_{\mathrm{CSB}}$ and $\mathcal{L}_{\mathrm{FSB}}$ break both the $\mathrm{SU}_{L}(3) \times$ $\mathrm{SU}_{R}(3)$ and diagonal $\mathrm{SU}(3)$ symmetry so that chiral symmetry cannot be conserved, these terms without derivatives yield no explicit contribution to the electromagnetic (EM) currents $J^{\mu}$ and at least in the adjoint representation of the $\mathrm{SU}(3)$ group the EM currents are conserved and of the same form as the chiral limit result $J_{\mathrm{CS}}^{\mu}$ to preserve the $U$-spin symmetry. However the derivative-dependent term in $\mathcal{L}_{\mathrm{FSB}}$ gives rise to the $U$-spin symmetry breaking conserved EM currents $J_{\mathrm{FSB}}^{\mu}$ so that $J^{\mu}=J_{\mathrm{CS}}^{\mu}+J_{\mathrm{FSB}}^{\mu}$.

Assuming that the hedgehog classical solution in the meson phase $U_{0}=e^{i \lambda_{i} \hat{r}_{i} \theta(r)}(i=1,2,3)$ is embedded in the $\mathrm{SU}(2)$ isospin subgroup of $\mathrm{SU}(3)$ and the Fock space in the quark phase is described by the $N_{c}$ valence quarks and the vacuum structure composed of quarks filling the negative energy sea, the chiral model generates the zero mode with the collective variable $A(t) \in \mathrm{SU}(3)$ by performing the slow rotation $U \rightarrow A U_{0} A^{\dagger}$ and $\psi \rightarrow A \psi$ on $\mathrm{SU}(3)$ group manifold. Given the spinning chiral model ansatz, the EM currents yield the magnetic moment operators $\hat{\mu}^{i}=\hat{\mu}^{i(3)}+\frac{1}{\sqrt{3}} \hat{\mu}^{i(8)}$, where $\hat{\mu}^{i(a)}=\hat{\mu}_{\mathrm{CS}}^{i(a)}+\hat{\mu}_{\mathrm{FSB}}^{i(a)}$ with

$$
\begin{aligned}
\hat{\mu}_{\mathrm{CS}}^{i(a)}= & -\mathcal{N} D_{a i}^{8}-\mathcal{N}^{\prime} d_{i p q} D_{a p}^{8} \hat{T}_{q}^{R}+\frac{N_{c}}{2 \sqrt{3}} \mathcal{M} D_{a 8}^{8} \hat{J}_{i}, \\
\hat{\mu}_{\mathrm{FSB}}^{i(a)}= & -\mathcal{P} D_{a i}^{8}\left(1-D_{88}^{8}\right)+\mathcal{Q} \frac{\sqrt{3}}{2} d_{i p q} D_{a p}^{8} D_{8 q}^{8} \\
& +\mathcal{R} D_{a 8}^{8} D_{8 i}^{8},
\end{aligned}
$$

where $\mathcal{M}, \mathcal{N}, \mathcal{N}^{\prime}, \mathcal{P}, \mathcal{Q}$, and $\mathcal{R}$ are the inertia parameters calculable in the chiral models $[23,24]$. Using the theorem that the tensor product of the Wigner $D$ functions can be decomposed into the sum of the single $D$ functions, the isovector and isoscalar parts of the operator $\hat{\mu}_{\mathrm{FSB}}^{i(a)}$ are then rewritten as

$$
\begin{aligned}
& \hat{\mu}_{\mathrm{FSB}}^{i(3)}=\mathcal{P}\left(-\frac{4}{5} D_{3 i}^{8}+\frac{1}{4} D_{3 i}^{10}+\frac{1}{4} D_{3 i}^{10}+\frac{3}{10} D_{3 i}^{27}\right)+\mathcal{Q}\left(\frac{3}{10} D_{3 i}^{8}-\frac{3}{10} D_{3 i}^{27}\right)+\mathcal{R}\left(\frac{1}{5} D_{3 i}^{8}+\frac{7}{10} D_{3 i}^{27}\right), \\
& \hat{\mu}_{\mathrm{FSB}}^{i(8)}=\mathcal{P}\left(-\frac{6}{5} D_{8 i}^{8}+\frac{9}{20} D_{8 i}^{27}\right)+\mathcal{Q}\left(-\frac{3}{10} D_{8 i}^{8}-\frac{9}{20} D_{8 i}^{27}\right)+\mathcal{R}\left(-\frac{1}{5} D_{8 i}^{8}+\frac{9}{20} D_{8 i}^{27}\right) .
\end{aligned}
$$

Here one notes that the $\mathbf{1 , 1 0}$, and $\overline{\mathbf{1 0}}$ irreducible representations (IRs) do not occur in the decuplet baryons while $\mathbf{1 0}$ and $\overline{\mathbf{1 0}}$ IRs appear together in the isovector channel of the baryon octet to conserve the hermiticity of the operator.

Using the above operator $\hat{\mu}^{i}$ together with the decuplet baryon wave function $\Phi_{B}^{\lambda}=\sqrt{\operatorname{dim}(\lambda)} D_{a b}^{\lambda}$ with the quantum numbers $a=\left(Y ; I, I_{3}\right)\left(Y\right.$, hypercharge; $I$, isospin) and $b=\left(Y_{R} ; J,-J_{3}\right)\left(Y_{R}\right.$, right hypercharge; $J$, spin $)$ and $\lambda$ the dimension of the representation, the baryon decuplet magnetic moments for $\mathbf{1 0}\left(J_{3}=3 / 2\right)$ and transition magnetic moments for $\mathbf{1 0}\left(J_{3}=1 / 2\right) \rightarrow \mathbf{8}\left(J_{3}=1 / 2\right)+\gamma$ have the following hyperfine structure: 


$$
\begin{aligned}
& \mu_{\Delta^{++}}=\frac{1}{8} \mathcal{M}+\frac{1}{2}\left(\mathcal{N}-\frac{1}{2 \sqrt{3}} \mathcal{N}^{\prime}\right)+\frac{3}{7} \mathcal{P}-\frac{3}{56} \mathcal{Q}-\frac{1}{14} \mathcal{R}, \\
& \mu_{\Delta^{+}}=\frac{1}{16} \mathcal{M}+\frac{1}{4}\left(\mathcal{N}-\frac{1}{2 \sqrt{3}} \mathcal{N}^{\prime}\right)+\frac{5}{21} \mathcal{P}+\frac{1}{84} \mathcal{Q}-\frac{1}{84} \mathcal{R}, \\
& \mu_{\Delta^{0}}=\frac{1}{21} \mathcal{P}+\frac{13}{168} \mathcal{Q}+\frac{1}{21} \mathcal{R}, \\
& \mu_{\Delta^{-}}=-\frac{1}{16} \mathcal{M}-\frac{1}{4}\left(\mathcal{N}-\frac{1}{2 \sqrt{3}} \mathcal{N}^{\prime}\right)-\frac{1}{7} \mathcal{P}+\frac{1}{7} \mathcal{Q}+\frac{3}{28} \mathcal{R}, \\
& \mu_{\Sigma^{*+}}=\frac{1}{16} \mathcal{M}+\frac{1}{4}\left(\mathcal{N}-\frac{1}{2 \sqrt{3}} \mathcal{N}^{\prime}\right)+\frac{19}{84} \mathcal{P}-\frac{17}{168} \mathcal{Q}-\frac{1}{42} \mathcal{R}, \\
& \mu_{\Sigma^{* 0}}=\frac{1}{84} \mathcal{P}-\frac{1}{84} \mathcal{Q}+\frac{1}{84} \mathcal{R}, \\
& \mu_{\Sigma^{*-}}=-\frac{1}{16} \mathcal{M}-\frac{1}{4}\left(\mathcal{N}-\frac{1}{2 \sqrt{3}} \mathcal{N}^{\prime}\right)-\frac{17}{84} \mathcal{P}+\frac{13}{168} \mathcal{Q}+\frac{1}{21} \mathcal{R}, \\
& \mu_{\Xi^{* 0}}=-\frac{1}{42} \mathcal{P}-\frac{17}{168} \mathcal{Q}-\frac{1}{42} \mathcal{R}, \\
& \mu_{\Xi^{*-}}=-\frac{1}{16} \mathcal{M}-\frac{1}{4}\left(\mathcal{N}-\frac{1}{2 \sqrt{3}} \mathcal{N}^{\prime}\right)-\frac{11}{42} \mathcal{P}+\frac{1}{84} \mathcal{Q}-\frac{1}{84} \mathcal{R}, \\
& \mu_{\Omega^{-}}=-\frac{1}{16} \mathcal{M}-\frac{1}{4}\left(\mathcal{N}-\frac{1}{2 \sqrt{3}} \mathcal{N}^{\prime}\right)-\frac{9}{28} \mathcal{P}-\frac{3}{56} \mathcal{Q}-\frac{1}{14} \mathcal{R}, \\
& \frac{1}{\sqrt{5}} \mu_{p \Delta^{+}}=\frac{1}{\sqrt{5}} \mu_{n \Delta^{0}}=-\frac{2}{15}\left(\mathcal{N}+\frac{2-\sqrt{2}}{8} \mathcal{N}^{\prime}\right)-\frac{4}{45} \mathcal{P}+\frac{7}{180} \mathcal{Q}+\frac{79}{2700} \mathcal{R}, \\
& \frac{1}{\sqrt{5}} \mu_{\Sigma^{+} \Sigma^{*+}}=\frac{2}{15}\left(\mathcal{N}+\frac{2-\sqrt{2}}{8} \mathcal{N}^{\prime}\right)+\frac{13}{90} \mathcal{P}+\frac{1}{180} \mathcal{Q}-\frac{1}{100} \mathcal{R}, \\
& \frac{1}{\sqrt{5}} \mu_{\Sigma^{0} \Sigma^{* 0}}=\frac{1}{15}\left(\mathcal{N}+\frac{2-\sqrt{2}}{8} \mathcal{N}^{\prime}\right)+\frac{7}{90} \mathcal{P}+\frac{1}{45} \mathcal{Q}+\frac{1}{90} \mathcal{R}, \\
& \frac{1}{\sqrt{5}} \mu_{\Sigma^{-} \Sigma^{*-}}=\frac{1}{90} \mathcal{P}+\frac{7}{180} \mathcal{Q}+\frac{29}{900} \mathcal{R}, \\
& \frac{1}{\sqrt{5}} \mu_{\Xi^{0} \Xi^{* 0}}=\frac{2}{15}\left(\mathcal{N}+\frac{2-\sqrt{2}}{8} \mathcal{N}^{\prime}\right)+\frac{7}{45} \mathcal{P}-\frac{1}{180} \mathcal{Q}+\frac{23}{2700} \mathcal{R}, \\
& \frac{1}{\sqrt{5}} \mu_{\Xi^{-} \Xi^{*-}}=\frac{1}{90} \mathcal{P}+\frac{7}{180} \mathcal{Q}+\frac{67}{2700} \mathcal{R}, \\
& \frac{1}{\sqrt{15}} \mu_{\Lambda \Sigma^{* 0}}=-\frac{1}{15}\left(\mathcal{N}+\frac{2-\sqrt{2}}{8} \mathcal{N}^{\prime}\right)-\frac{1}{18} \mathcal{P}+\frac{1}{45} \mathcal{Q}+\frac{11}{1350} \mathcal{R} \text {. }
\end{aligned}
$$

Here the coefficients are solely given by the SU(3) group structure of the chiral models and the physical information such as decay constants and masses are included in the above inertia parameters, such as $\mathcal{M}, \mathcal{N}$, and so on. Note that the $\mathrm{SU}(3)$ group structure in the coefficients is generic property shared by the chiral models which exploit the hedgehog ansatz solution corresponding to the little group $\mathrm{SU}(2) \times \mathbf{Z}_{2}$ [25]. In the chiral perturbation theory [8] and in the $1 / N_{c}$ expansion of QCD [14-16] to which the hedgehog ansatz does not apply, one can thus see the coefficients different from those in (2.4) even though the
$\mathrm{SU}$ (3) flavor group is used in the theory. Now, it seems appropriate to comment on the $1 / N_{c}$ expansion [25-28]. In the above relations (2.4), the inertia parameters $\mathcal{N}, \mathcal{N}^{\prime}$, $\mathcal{P}, \mathcal{Q}$, and $\mathcal{R}$ are of order $N_{c}$ while $\mathcal{M}$ is of order $N_{c}^{-1}$. However, since the inertia parameter $\mathcal{M}$ is multiplied by an explicit factor $N_{c}$ in (2.2), the terms with $\mathcal{M}$ are of order $N_{c}^{0}$. (For details of further $1 / N_{c}$, see $[25,28]$.)

In the $\mathrm{SU}(3)$ flavor symmetric limit with the chiral symmetry breaking masses $m_{u}=m_{d}=m_{s}, m_{K}=m_{\pi}$ and decay constants $f_{K}=f_{\pi}$, the magnetic moments of the decuplet baryons are simply given by [29] 


$$
\mu_{B}=Q_{\mathrm{EM}}\left(\frac{1}{16} \mathcal{M}+\frac{1}{4}\left(\mathcal{N}-\frac{1}{2 \sqrt{3}} \mathcal{N}^{\prime}\right)\right)
$$

where $Q_{\mathrm{EM}}$ is the EM charge. Here one notes that in the chiral model in the adjoint representation the prediction of the baryon magnetic moments with the chiral symmetry is the same as that with the SU(3) flavor symmetry since the mass-dependent term in $\mathcal{L}_{\mathrm{CSB}}$ and $\mathcal{L}_{\mathrm{FSB}}$ do not yield any contribution to $J_{\mathrm{FSB}}^{\mu}$ so that there are no terms with $\mathcal{P}, \mathcal{Q}$, and $\mathcal{R}$ in (2.4). Because of the degenerate $d$ - and $s$-flavor charges in the SU(3) EM charge operator $\hat{Q}_{\mathrm{EM}}$, the chiral model possesses the $U$-spin symmetry relations in the baryon decuplet magnetic moments, similar to those in the octet baryons [30]

$$
\begin{gathered}
\mu_{\Delta^{-}}=\mu_{\Sigma^{*-}}=\mu_{\Xi^{*-}}=\mu_{\Omega^{-}}, \quad \mu_{\Delta^{0}}=\mu_{\Sigma^{* 0}}=\mu_{\Xi^{* 0}}, \\
\mu_{\Delta^{+}}=\mu_{\Sigma^{*+}},
\end{gathered}
$$

which are a subset of the stronger symmetry relations (2.5).
Next, since the SU(3) FSB quark masses do not affect the magnetic moments of the baryon decuplet in the adjoint representation of the chiral model, in the more general $\mathrm{SU}(3)$ flavor symmetry broken case with $m_{u}=m_{d} \neq m_{s}$, $m_{\pi} \neq m_{K}$ and $f_{\pi} \neq f_{K}$, the decuplet baryon magnetic moments with all the inertia parameters satisfy the following symmetric sum rules:

$$
\begin{gathered}
\mu_{\Sigma^{* 0}}=\frac{1}{2} \mu_{\Sigma^{*+}}+\frac{1}{2} \mu_{\Sigma^{*-}}, \\
\mu_{\Delta^{-}}+\mu_{\Delta^{++}}=\mu_{\Delta^{0}}+\mu_{\Delta^{+}}, \quad \sum_{B \in \text { decuplet }} \mu_{B}=0 .
\end{gathered}
$$

Now, in order to predict baryon decuplet magnetic moments we proceed to derive sum rules for the baryon magnetic moments in terms of the experimentally known baryon magnetic moments. To do this, we first consider the baryon octet magnetic moments of the form

$$
\begin{aligned}
\mu_{p} & =\frac{1}{10} \mathcal{M}+\frac{4}{15}\left(\mathcal{N}+\frac{1}{2} \mathcal{N}^{\prime}\right)+\frac{8}{45} \mathcal{P}-\frac{2}{45} \mathcal{Q}-\frac{1}{45} \mathcal{R} \\
\mu_{n} & =\frac{1}{20} \mathcal{M}-\frac{1}{5}\left(\mathcal{N}+\frac{1}{2} \mathcal{N}^{\prime}\right)-\frac{1}{9} \mathcal{P}+\frac{7}{90} \mathcal{Q}+\frac{1}{45} \mathcal{R} \\
\mu_{\Sigma^{+}} & =\frac{1}{10} \mathcal{M}+\frac{4}{15}\left(\mathcal{N}+\frac{1}{2} \mathcal{N}^{\prime}\right)+\frac{13}{45} \mathcal{P}-\frac{1}{45} \mathcal{Q}-\frac{2}{45} \mathcal{R} \\
\mu_{\Sigma^{0}} & =-\frac{1}{40} \mathcal{M}+\frac{1}{10}\left(\mathcal{N}+\frac{1}{2} \mathcal{N}^{\prime}\right)+\frac{11}{90} \mathcal{P}+\frac{1}{36} \mathcal{Q}+\frac{1}{45} \mathcal{R} \\
\mu_{\Sigma^{-}} & =-\frac{3}{20} \mathcal{M}-\frac{1}{15}\left(\mathcal{N}+\frac{1}{2} \mathcal{N}^{\prime}\right)-\frac{2}{45} \mathcal{P}+\frac{7}{90} \mathcal{Q}+\frac{4}{45} \mathcal{R} \\
\mu_{\Xi^{0}} & =\frac{1}{20} \mathcal{M}-\frac{1}{5}\left(\mathcal{N}+\frac{1}{2} \mathcal{N}^{\prime}\right)-\frac{11}{45} \mathcal{P}-\frac{1}{45} \mathcal{Q}+\frac{1}{45} \mathcal{R} \\
\mu_{\Xi^{-}} & =-\frac{3}{20} \mathcal{M}-\frac{1}{15}\left(\mathcal{N}+\frac{1}{2} \mathcal{N}^{\prime}\right)-\frac{4}{45} \mathcal{P}-\frac{2}{45} \mathcal{Q}-\frac{4}{45} \mathcal{R} \\
\mu_{\Lambda} & =\frac{1}{40} \mathcal{M}-\frac{1}{10}\left(\mathcal{N}+\frac{1}{2} \mathcal{N}^{\prime}\right)-\frac{1}{10} \mathcal{P}-\frac{1}{20} \mathcal{Q}
\end{aligned}
$$

Since we have effectively five inertia parameters $\mathcal{M}, \mathcal{N}+\frac{1}{2} \mathcal{N}^{\prime}, \mathcal{P}, \mathcal{Q}$, and $\mathcal{R}$, we can derive sum rules for three magnetic moments $\mu_{\Sigma^{0}}, \mu_{\Lambda}$, and $\mu_{\Xi^{-}}$in terms of five experimentally known octet magnetic moments, $\mu_{p}, \mu_{n}, \mu_{\Sigma^{+}}, \mu_{\Sigma^{-}}$, and $\mu_{\Xi^{0}}$ as follows:

$$
\begin{gathered}
\mu_{\Sigma^{0}}=\frac{1}{2} \mu_{\Sigma^{+}}+\frac{1}{2} \mu_{\Sigma^{-}}, \quad \mu_{\Xi^{-}}=-\frac{1}{3} \mu_{n}-\frac{8}{3} \mu_{\Sigma^{+}}-\frac{5}{3} \mu_{\Sigma^{-}}-\frac{7}{3} \mu_{\Xi^{0}}, \\
\mu_{\Lambda}=-\mu_{p}-\frac{2}{3} \mu_{n}+\frac{7}{6} \mu_{\Sigma^{+}}+\frac{1}{6} \mu_{\Sigma^{-}}+\frac{4}{3} \mu_{\Xi^{0}} .
\end{gathered}
$$

Using the above sum rules we can predict the magnetic moments as in Table I. One notes that the value of $\mu_{\Lambda}$ is comparable to the experimental data $\mu_{\Lambda}^{\exp }=-0.61$, while the value of $\mu_{\Xi^{-}}$is not so comparable to $\mu_{\Xi^{-}}^{\exp }=-0.65$.

Similarly we can derive sum rules for the decuplet magnetic moments in terms of six experimentally known magnetic moments, $\mu_{p}, \mu_{n}, \mu_{\Sigma^{+}}, \mu_{\Sigma^{-}}, \mu_{\Xi^{0}}$, and $\mu_{\Delta^{++}}$to arrive at 


$$
\begin{aligned}
& \mu_{\Delta^{+}}=\frac{5}{28} \mu_{p}+\frac{55}{168} \mu_{n}+\frac{5}{42} \mu_{\Sigma^{+}}+\frac{25}{84} \mu_{\Sigma^{-}}-\frac{5}{168} \mu_{\Xi^{0}}+\frac{1}{2} \mu_{\Delta^{++}}, \\
& \mu_{\Delta^{0}}=\frac{5}{14} \mu_{p}+\frac{55}{84} \mu_{n}+\frac{5}{21} \mu_{\Sigma^{+}}+\frac{25}{42} \mu_{\Sigma^{-}}-\frac{5}{84} \mu_{\Xi^{0}}, \\
& \mu_{\Delta^{-}}=\frac{15}{28} \mu_{p}+\frac{55}{56} \mu_{n}+\frac{5}{14} \mu_{\Sigma^{+}}+\frac{25}{28} \mu_{\Sigma^{-}}-\frac{5}{56} \mu_{\Xi^{0}}-\frac{1}{2} \mu_{\Delta^{++}}, \\
& \mu_{\Sigma^{*+}}=-\frac{25}{14} \mu_{p}-\frac{235}{168} \mu_{n}+\frac{215}{84} \mu_{\Sigma^{+}}+\frac{65}{84} \mu_{\Sigma^{-}}+\frac{365}{168} \mu_{\Xi^{0}}+\frac{1}{2} \mu_{\Delta^{++}} \text {, } \\
& \mu_{\Sigma^{* 0}}=-\frac{15}{28} \mu_{p}-\frac{5}{14} \mu_{n}+\frac{25}{28} \mu_{\Sigma^{+}}+\frac{5}{14} \mu_{\Sigma^{-}}+\frac{5}{7} \mu_{\Xi^{0}}, \\
& \mu_{\Sigma^{*-}}=\frac{5}{7} \mu_{p}+\frac{115}{168} \mu_{n}-\frac{65}{84} \mu_{\Sigma^{+}}-\frac{5}{84} \mu_{\Sigma^{-}}-\frac{125}{168} \mu_{\Xi^{0}}-\frac{1}{2} \mu_{\Delta^{++}}, \\
& \mu_{\Xi^{* 0}}=-\frac{10}{7} \mu_{p}-\frac{115}{84} \mu_{n}+\frac{65}{42} \mu_{\Sigma^{+}}+\frac{5}{42} \mu_{\Sigma^{-}}+\frac{125}{84} \mu_{\Xi^{0}}, \\
& \mu_{\Xi^{*-}}=\frac{25}{28} \mu_{p}+\frac{65}{168} \mu_{n}-\frac{40}{21} \mu_{\Sigma^{+}}-\frac{85}{84} \mu_{\Sigma^{-}}-\frac{235}{168} \mu_{\Xi^{0}}-\frac{1}{2} \mu_{\Delta^{++}}, \\
& \mu_{\Omega^{-}}=\frac{15}{14} \mu_{p}+\frac{5}{56} \mu_{n}-\frac{85}{28} \mu_{\Sigma^{+}}-\frac{55}{28} \mu_{\Sigma^{-}}-\frac{115}{56} \mu_{\Xi^{0}}-\frac{1}{2} \mu_{\Delta^{++}}, \\
& \frac{1}{\sqrt{5}} \mu_{p \Delta^{+}}=\frac{1}{\sqrt{5}} \mu_{n \Delta^{0}}=\left(-\frac{1}{189}+\frac{17 \sqrt{2}}{140}-\frac{17 \sqrt{3}}{210}-\frac{17 \sqrt{6}}{420}\right) \mu_{p}+\left(\frac{173}{567}+\frac{\sqrt{2}}{168}-\frac{\sqrt{3}}{252}-\frac{\sqrt{6}}{504}\right) \mu_{n} \\
& +\left(\frac{3383}{11340}-\frac{\sqrt{2}}{420}+\frac{\sqrt{3}}{630}+\frac{\sqrt{6}}{1260}\right) \mu_{\Sigma^{+}}+\left(\frac{2189}{11340}-\frac{13 \sqrt{2}}{420}+\frac{13 \sqrt{3}}{630}+\frac{13 \sqrt{6}}{1260}\right) \mu_{\Sigma^{-}} \\
& +\left(\frac{2131}{11340}-\frac{73 \sqrt{2}}{840}+\frac{73 \sqrt{3}}{1260}+\frac{73 \sqrt{6}}{2520}\right) \mu_{\Xi^{0}}+\left(-\frac{1}{5}-\frac{\sqrt{2}}{10}+\frac{\sqrt{3}}{15}+\frac{\sqrt{6}}{30}\right) \mu_{\Delta^{++}}, \\
& \frac{1}{\sqrt{5}} \mu_{\Sigma^{+} \Sigma^{*+}}=\left(-\frac{83}{630}-\frac{17 \sqrt{2}}{140}+\frac{17 \sqrt{3}}{210}+\frac{17 \sqrt{6}}{420}\right) \mu_{p}+\left(-\frac{1}{378}-\frac{\sqrt{2}}{168}+\frac{\sqrt{3}}{252}+\frac{\sqrt{6}}{504}\right) \mu_{n} \\
& +\left(\frac{131}{756}+\frac{\sqrt{2}}{420}-\frac{\sqrt{3}}{630}-\frac{\sqrt{6}}{1260}\right) \mu_{\Sigma^{+}}+\left(\frac{107}{756}+\frac{13 \sqrt{2}}{420}-\frac{13 \sqrt{3}}{630}-\frac{13 \sqrt{6}}{1260}\right) \mu_{\Sigma^{-}} \\
& +\left(-\frac{589}{3780}+\frac{73 \sqrt{2}}{840}-\frac{73 \sqrt{3}}{1260}-\frac{73 \sqrt{6}}{2520}\right) \mu_{\Xi^{0}}+\left(\frac{1}{5}+\frac{\sqrt{2}}{10}-\frac{\sqrt{3}}{15}-\frac{\sqrt{6}}{30}\right) \mu_{\Delta^{++}}, \\
& \frac{1}{\sqrt{5}} \mu_{\Sigma^{0} \Sigma^{* 0}}=\left(\frac{11}{140}-\frac{17 \sqrt{2}}{280}+\frac{17 \sqrt{3}}{420}+\frac{17 \sqrt{6}}{840}\right) \mu_{p}+\left(\frac{9}{56}-\frac{\sqrt{2}}{336}+\frac{\sqrt{3}}{504}+\frac{\sqrt{6}}{1008}\right) \mu_{n}+\left(\frac{19}{140}+\frac{\sqrt{2}}{840}-\frac{\sqrt{3}}{1260}-\frac{\sqrt{6}}{2520}\right) \mu_{\Sigma^{+}} \\
& +\left(\frac{37}{140}+\frac{13 \sqrt{2}}{840}-\frac{13 \sqrt{3}}{1260}-\frac{13 \sqrt{6}}{2520}\right) \mu_{\Sigma^{-}}+\left(-\frac{13}{280}+\frac{73 \sqrt{2}}{1680}-\frac{73 \sqrt{3}}{2520}-\frac{73 \sqrt{6}}{5040}\right) \mu_{\Xi^{0}} \\
& +\left(\frac{1}{10}+\frac{\sqrt{2}}{20}-\frac{\sqrt{3}}{30}-\frac{\sqrt{6}}{60}\right) \mu_{\Delta^{++}} \\
& \frac{1}{\sqrt{5}} \mu_{\Sigma^{-} \Sigma^{*-}}=\frac{13}{45} \mu_{p}+\frac{35}{108} \mu_{n}+\frac{53}{540} \mu_{\Sigma^{+}}+\frac{209}{540} \mu_{\Sigma^{-}}+\frac{17}{270} \mu_{\Xi^{0}}, \\
& \frac{1}{\sqrt{5}} \mu_{\Xi^{0} \Xi^{* 0}}=\left(-\frac{667}{945}-\frac{17 \sqrt{2}}{140}+\frac{17 \sqrt{3}}{210}+\frac{17 \sqrt{6}}{420}\right) \mu_{p}+\left(-\frac{451}{1134}-\frac{\sqrt{2}}{168}+\frac{\sqrt{3}}{252}+\frac{\sqrt{6}}{504}\right) \mu_{n} \\
& +\left(\frac{2759}{2268}+\frac{\sqrt{2}}{420}-\frac{\sqrt{3}}{630}-\frac{\sqrt{6}}{1260}\right) \mu_{\Sigma^{+}}+\left(\frac{1385}{2268}+\frac{13 \sqrt{2}}{420}-\frac{13 \sqrt{3}}{630}-\frac{13 \sqrt{6}}{1260}\right) \mu_{\Sigma^{-}} \\
& +\left(\frac{8033}{11340}+\frac{73 \sqrt{2}}{840}-\frac{73 \sqrt{3}}{1260}-\frac{73 \sqrt{6}}{2520}\right) \mu_{\Xi^{0}}+\left(\frac{1}{5}+\frac{\sqrt{2}}{10}-\frac{\sqrt{3}}{15}-\frac{\sqrt{6}}{30}\right) \mu_{\Delta^{++}},
\end{aligned}
$$


TABLE I. The magnetic moments, their flavor components, and strange form factors of the decuplet and octet baryons. The quantities used as input parameters are indicated by $*$.

\begin{tabular}{lccrrr}
\hline \hline$B$ & $\mu_{B}$ & $\mu_{B}^{(s)}$ & $\mu_{B}^{(u)}$ & $\mu_{B}^{(d)}$ & $F_{2 B}^{(s)}$ \\
\hline$\Delta^{++}$ & $4.52^{*}$ & 0.07 & 4.10 & 0.35 & -0.21 \\
$\Delta^{+}$ & 2.12 & 0.07 & 2.50 & -0.45 & -0.21 \\
$\Delta^{0}$ & -0.29 & 0.07 & 0.89 & -1.25 & -0.21 \\
$\Delta^{-}$ & -2.69 & 0.07 & -0.71 & -2.05 & -0.21 \\
$\Sigma^{*+}$ & 2.63 & -0.49 & 2.84 & 0.28 & 0.47 \\
$\Sigma^{* 0}$ & 0.08 & -0.49 & 1.14 & -0.57 & 0.47 \\
$\Sigma^{*-}$ & -2.48 & -0.49 & -0.57 & -1.42 & 0.47 \\
$\Xi^{* 0}$ & 0.44 & -1.15 & 1.38 & 0.21 & 1.45 \\
$\Xi^{*-}$ & -2.27 & -1.15 & -0.43 & -0.69 & 1.45 \\
$\Omega^{-}$ & -2.06 & -1.92 & -0.29 & 0.15 & 2.76 \\
$p$ & $2.79^{*}$ & -0.25 & 2.94 & 0.10 & 0.75 \\
$n$ & $-1.91^{*}$ & -0.25 & -0.19 & -1.47 & 0.75 \\
$\Sigma^{+}$ & $2.46^{*}$ & -0.11 & 2.72 & -0.15 & -0.67 \\
$\Sigma^{0}$ & 0.65 & -0.11 & 1.52 & -0.76 & -0.67 \\
$\Sigma^{-}$ & $-1.16^{*}$ & -0.11 & 0.31 & -1.36 & -0.67 \\
$\Xi^{0}$ & $-1.25^{*}$ & -1.32 & 0.25 & -0.18 & 1.95 \\
$\Xi^{-}$ & -1.07 & -1.32 & 0.37 & -0.12 & 1.95 \\
$\Lambda$ & -0.51 & -0.88 & 0.75 & -0.38 & 1.64 \\
\hline \hline
\end{tabular}

$$
\begin{aligned}
\frac{1}{\sqrt{5}} \mu_{\Xi^{-} \Xi^{*-}}= & \frac{49}{135} \mu_{p}+\frac{125}{324} \mu_{n}-\frac{181}{1620} \mu_{\Sigma^{+}}+\frac{407}{1620} \mu_{\Sigma^{-}}-\frac{109}{810} \mu_{\Xi^{0}}, \\
\frac{1}{\sqrt{15}} \mu_{\Lambda \Sigma^{* 0}}= & \left.\frac{215}{756}+\frac{17 \sqrt{2}}{280}-\frac{17 \sqrt{3}}{420}-\frac{17 \sqrt{6}}{840}\right) \mu_{p}+\left(\frac{1399}{4536}+\frac{\sqrt{2}}{336}-\frac{\sqrt{3}}{504}-\frac{\sqrt{6}}{1008}\right) \mu_{n} \\
& +\left(-\frac{3751}{11340}-\frac{\sqrt{2}}{840}+\frac{\sqrt{3}}{1260}+\frac{\sqrt{6}}{2520}\right) \mu_{\Sigma^{+}}+\left(-\frac{1093}{11340}-\frac{13 \sqrt{2}}{840}+\frac{13 \sqrt{3}}{1260}+\frac{13 \sqrt{6}}{2520}\right) \mu_{\Sigma^{-}} \\
& +\left(-\frac{5779}{22680}-\frac{73 \sqrt{2}}{1680}+\frac{73 \sqrt{3}}{2520}+\frac{73 \sqrt{6}}{5040}\right) \mu_{\Xi^{0}}+\left(-\frac{1}{10}-\frac{\sqrt{2}}{20}+\frac{\sqrt{3}}{30}+\frac{\sqrt{6}}{60}\right) \mu_{\Delta^{++}} .
\end{aligned}
$$

Here we have used the additional magnetic moment $\mu_{\Delta^{++}}$ since we have six inertia parameters $\mathcal{M}, \mathcal{N}, \mathcal{N}^{\prime}, \mathcal{P}, \mathcal{Q}$, and $\mathcal{R}$. We list the predictions for the magnetic moments of the decuplet and octet baryons in Table I, and those for the decuplet-to-octet transition magnetic moments in Table II, by using their sum rules (2.10). Here note that our prediction of $\mu_{\Omega^{-}}$is comparable to its experimental value $\mu_{\Omega^{-}}^{\exp }=-1.94$. The predicted values of the decupletto-octet transition magnetic moments in Table II are also comparable to the previous ones obtained by using the chiral quark soliton model [17].

TABLE II. The transition magnetic moments and their flavor components for $\mathbf{1 0}\left(J_{3}=1 / 2\right) \rightarrow$ $\mathbf{8}\left(J_{3}=1 / 2\right)+\gamma$.

\begin{tabular}{lrrrr}
\hline \hline$B_{8} B_{10}$ & $\mu_{B_{8} B_{10}}$ & $\mu_{B_{8} B_{10}}^{(s)}$ & $\mu_{B_{8} B_{10}}^{(u)}$ & $\mu_{B_{8} B_{10}}^{(d)}$ \\
\hline$p \Delta^{+}$ & -2.76 & -0.54 & -0.76 & -1.46 \\
$n \Delta^{0}$ & -2.76 & -0.54 & -0.76 & -1.46 \\
$\Sigma^{+} \Sigma^{*+}$ & 2.24 & 0.13 & 2.58 & -0.47 \\
$\Sigma^{0} \Sigma^{* 0}$ & 1.01 & 0.13 & 1.76 & -0.88 \\
$\Sigma^{-} \Sigma^{*-}$ & -0.22 & 0.13 & 0.94 & -1.29 \\
$\Xi^{0} \Xi^{* 0}$ & 2.46 & 0.19 & 2.72 & -0.45 \\
$\Xi^{-} \Xi^{*-}$ & -0.27 & 0.19 & 0.90 & -1.36 \\
$\Lambda \Sigma^{* 0}$ & -2.46 & -0.54 & -0.56 & -1.36 \\
\hline \hline
\end{tabular}




\section{STRANGE FLAVOR MAGNETIC MOMENTS OF BARYON DECUPLET}

In the SU(3) flavor symmetry broken case we decompose the EM currents into three pieces $J^{\mu}=J^{\mu(u)}+J^{\mu(d)}+J^{\mu(s)}$ where the $q$-flavor currents $J^{\mu(q)}=J_{\mathrm{CS}}^{\mu(q)}+J_{\mathrm{FSB}}^{\mu(q)}$ are given by substituting the charge operator $\hat{Q}$ with the $q$-flavor charge operator $\hat{Q}_{q}$

$$
\begin{aligned}
J_{\mathrm{CS}}^{\mu(q)}= & \bar{\psi} \gamma^{\mu} \hat{Q}_{q} \psi \Theta_{B}+\left(-\frac{i}{2} f_{\pi}^{2} \operatorname{tr}\left(\hat{Q}_{q} l^{\mu}\right)+\frac{i}{8 e^{2}} \operatorname{tr}\left[\hat{Q}_{q}, l^{\nu}\right]\left[l^{\mu}, l^{\nu}\right]+U \leftrightarrow U^{\dagger}\right) \bar{\Theta}_{B} \\
& +\frac{N_{c}}{48 \pi^{2}} \epsilon^{\mu \nu \alpha \beta} \operatorname{tr}\left(\hat{Q}_{q} l_{\nu} l_{\alpha} l_{\beta}-U \leftrightarrow U^{\dagger}\right) \bar{\Theta}_{B}, \\
J_{\mathrm{FSB}}^{\mu(q)}= & -\frac{i}{12} f_{\pi}^{2}\left(\chi^{2}-1\right) \operatorname{tr}\left(\left(1-\sqrt{3} \lambda_{8}\right)\left(U \hat{Q}_{q} l^{\mu}+l^{\mu} \hat{Q}_{q} U^{\dagger}\right)+U \leftrightarrow U^{\dagger}\right) \bar{\Theta}_{B}
\end{aligned}
$$

to obtain the baryon magnetic moments and transition magnetic moments in the $s$-flavor channel

$$
\begin{aligned}
\mu_{\Delta}^{(s)} & =-\frac{7}{48} \mathcal{M}+\frac{1}{12}\left(\mathcal{N}-\frac{1}{2 \sqrt{3}} \mathcal{N}^{\prime}\right)+\frac{2}{21} \mathcal{P}+\frac{5}{168} \mathcal{Q}+\frac{1}{84} \mathcal{R} \\
\mu_{\Sigma^{*}}^{(s)} & =-\frac{1}{6} \mathcal{M}+\frac{1}{126} \mathcal{P}-\frac{1}{126} \mathcal{Q}+\frac{1}{126} \mathcal{R} \\
\mu_{\Xi^{*}}^{(s)} & =-\frac{3}{16} \mathcal{M}-\frac{1}{12}\left(\mathcal{N}-\frac{1}{2 \sqrt{3}} \mathcal{N}^{\prime}\right)-\frac{2}{21} \mathcal{P}-\frac{5}{168} \mathcal{Q}-\frac{1}{84} \mathcal{R} \\
\mu_{\Omega}^{(s)} & =-\frac{5}{24} \mathcal{M}-\frac{1}{6}\left(\mathcal{N}-\frac{1}{2 \sqrt{3}} \mathcal{N}^{\prime}\right)-\frac{3}{14} \mathcal{P}-\frac{1}{28} \mathcal{Q}-\frac{1}{21} \mathcal{R} \\
\mu_{N}^{(s)} & =-\frac{7}{60} \mathcal{M}+\frac{1}{45}\left(\mathcal{N}+\frac{1}{2} \mathcal{N}^{\prime}\right)+\frac{1}{45} \mathcal{P}+\frac{1}{90} \mathcal{Q} \\
\mu_{\Sigma}^{(s)} & =-\frac{11}{60} \mathcal{M}+\frac{1}{15}\left(\mathcal{N}+\frac{1}{2} \mathcal{N}^{\prime}\right)+\frac{11}{135} \mathcal{P}+\frac{1}{54} \mathcal{Q}+\frac{2}{135} \mathcal{R}, \\
\mu_{\Xi}^{(s)} & =-\frac{1}{5} \mathcal{M}-\frac{4}{45}\left(\mathcal{N}+\frac{1}{2} \mathcal{N}^{\prime}\right)-\frac{1}{9} \mathcal{P}-\frac{1}{45} \mathcal{Q}-\frac{1}{45} \mathcal{R} \\
\mu_{\Lambda}^{(s)} & =-\frac{3}{20} \mathcal{M}-\frac{1}{15}\left(\mathcal{N}+\frac{1}{2} \mathcal{N}^{\prime}\right)-\frac{1}{15} \mathcal{P}-\frac{1}{30} \mathcal{Q}, \\
\frac{1}{\sqrt{5}} \mu_{N \Delta}^{(s)} & =-\frac{1}{6 \sqrt{5}} \mathcal{M}, \\
\frac{1}{\sqrt{5}} \mu_{\Sigma \Sigma^{*}}^{(s)} & =-\frac{1}{6 \sqrt{5}} \mathcal{M}+\frac{2}{45}\left(\mathcal{N}+\frac{2-\sqrt{2}}{8} \mathcal{N}\right)+\frac{7}{135} \mathcal{P}+\frac{2}{135} \mathcal{Q}+\frac{1}{135} \mathcal{R}, \\
\frac{1}{\sqrt{5}} \mu_{\Xi \Xi^{*}}^{(s)} & =-\frac{1}{6 \sqrt{5}} \mathcal{M}+\frac{2}{45}\left(\mathcal{N}+\frac{2-\sqrt{2}}{8} \mathcal{N}\right)+\frac{1}{18} \mathcal{P}+\frac{1}{90} \mathcal{Q}+\frac{1}{90} \mathcal{R}, \\
\frac{1}{\sqrt{15}} \mu_{\Lambda \Sigma^{* 0}}^{(s)} & =-\frac{1}{6 \sqrt{15}} \mathcal{M} .
\end{aligned}
$$

Similarly, in the $u$ - and $d$-flavor channels of the adjoint representation we obtain the baryon magnetic moments and transition magnetic moments (A1) in the Appendix. Here one notes that in general all the baryon decuplet and octet magnetic moments fulfill the model independent relations in the $u$-and $d$-flavor components and the $I$-spin symmetry of the isomultiplets with the same strangeness in the $s$-flavor channel

$$
\mu_{B}^{(d)}=\frac{Q_{d}}{Q_{u}} \mu_{\bar{B}}^{(u)}, \quad \mu_{B}^{(s)}=\mu_{\bar{B}}^{(s)}
$$

with $\bar{B}$ being the isospin conjugate baryon in the isomultiplets of the baryon.

As in the previous sum rules of the magnetic moments (2.9) and (2.10), we find the sum rules of the strange components of the magnetic moments and transition magnetic moments in terms of the six experimentally known magnetic moments: 


$$
\begin{aligned}
& \mu_{\Delta}^{(s)}=-\frac{13}{84} \mu_{p}-\frac{115}{504} \mu_{n}-\frac{83}{126} \mu_{\Sigma^{+}}+\frac{47}{252} \mu_{\Sigma^{-}}-\frac{463}{504} \mu_{\Xi^{0}}+\frac{1}{6} \mu_{\Delta^{++}}, \\
& \mu_{\Sigma^{*}}^{(s)}=-\frac{29}{42} \mu_{p}-\frac{50}{63} \mu_{n}-\frac{23}{126} \mu_{\Sigma^{+}}+\frac{8}{63} \mu_{\Sigma^{-}}-\frac{26}{63} \mu_{\Xi^{0}}, \\
& \mu_{\Xi^{*}}^{(s)}=-\frac{43}{84} \mu_{p}-\frac{445}{504} \mu_{n}-\frac{113}{126} \mu_{\Sigma^{+}}-\frac{103}{252} \mu_{\Sigma^{-}}-\frac{433}{504} \mu_{\Xi^{0}}-\frac{1}{6} \mu_{\Delta^{++}}, \\
& \mu_{\Omega}^{(s)}=\frac{8}{21} \mu_{p}-\frac{125}{252} \mu_{n}-\frac{353}{126} \mu_{\Sigma^{+}}-\frac{179}{126} \mu_{\Sigma^{-}}-\frac{569}{252} \mu_{\Xi^{0}}-\frac{1}{3} \mu_{\Delta^{++}}, \\
& \mu_{N}^{(s)}=-\frac{2}{9} \mu_{n}-\frac{7}{9} \mu_{\Sigma^{+}}-\frac{1}{9} \mu_{\Sigma^{-}}-\frac{8}{9} \mu_{\Xi^{0}}, \\
& \mu_{\Sigma}^{(s)}=-\frac{1}{3} \mu_{p}-\frac{5}{9} \mu_{n}-\frac{4}{9} \mu_{\Sigma^{+}}+\frac{2}{9} \mu_{\Sigma^{-}}-\frac{8}{9} \mu_{\Xi^{0}}, \\
& \mu_{\Xi}^{(s)}=-\frac{1}{3} \mu_{p}-\frac{2}{3} \mu_{n}-\frac{5}{3} \mu_{\Sigma^{+}}-\frac{2}{3} \mu_{\Sigma^{-}}-\frac{4}{3} \mu_{\Xi^{0}}, \\
& \mu_{\Lambda}^{(s)}=-\mu_{p}-\mu_{n}, \\
& \frac{1}{\sqrt{5}} \mu_{N \Delta}^{(s)}=-\frac{\sqrt{5}}{15} \mu_{p}-\frac{\sqrt{5}}{9} \mu_{n}-\frac{7 \sqrt{5}}{45} \mu_{\Sigma^{+}}-\frac{\sqrt{5}}{45} \mu_{\Sigma^{-}}-\frac{8 \sqrt{5}}{45} \mu_{\Xi^{0}}, \\
& \frac{1}{\sqrt{5}} \mu_{\Sigma \Sigma^{*}}^{(s)}=\left(\frac{11}{210}-\frac{17 \sqrt{2}}{420}+\frac{17 \sqrt{3}}{630}-\frac{\sqrt{5}}{15}+\frac{17 \sqrt{6}}{1260}\right) \mu_{p}+\left(\frac{3}{28}-\frac{\sqrt{2}}{504}+\frac{\sqrt{3}}{756}-\frac{\sqrt{5}}{9}+\frac{\sqrt{6}}{1512}\right) \mu_{n} \\
& +\left(\frac{19}{210}+\frac{\sqrt{2}}{1260}-\frac{\sqrt{3}}{1890}-\frac{7 \sqrt{5}}{45}-\frac{\sqrt{6}}{3780}\right) \mu_{\Sigma^{+}}+\left(\frac{37}{210}+\frac{13 \sqrt{2}}{1260}-\frac{13 \sqrt{3}}{1890}-\frac{\sqrt{5}}{45}-\frac{13 \sqrt{6}}{3780}\right) \mu_{\Sigma^{-}} \\
& +\left(-\frac{13}{420}+\frac{73 \sqrt{2}}{2520}-\frac{73 \sqrt{3}}{3780}-\frac{8 \sqrt{5}}{45}-\frac{73 \sqrt{6}}{7560}\right) \mu_{\Xi^{0}}+\left(\frac{1}{15}+\frac{\sqrt{2}}{30}-\frac{\sqrt{3}}{45}-\frac{\sqrt{6}}{90}\right) \mu_{\Delta^{++}}, \\
& \frac{1}{\sqrt{5}} \mu_{\Xi \Xi^{*}}^{(s)}=\left(-\frac{4}{35}-\frac{17 \sqrt{2}}{420}+\frac{17 \sqrt{3}}{630}-\frac{\sqrt{5}}{15}+\frac{17 \sqrt{6}}{1260}\right) \mu_{p}+\left(-\frac{1}{252}-\frac{\sqrt{2}}{504}+\frac{\sqrt{3}}{756}-\frac{\sqrt{5}}{9}+\frac{\sqrt{6}}{1512}\right) \mu_{n} \\
& +\left(\frac{116}{315}+\frac{\sqrt{2}}{1260}-\frac{\sqrt{3}}{1890}-\frac{7 \sqrt{5}}{45}-\frac{\sqrt{6}}{3780}\right) \mu_{\Sigma^{+}}+\left(\frac{181}{630}+\frac{13 \sqrt{2}}{1260}-\frac{13 \sqrt{3}}{1890}-\frac{\sqrt{5}}{45}-\frac{13 \sqrt{6}}{3780}\right) \mu_{\Sigma^{-}} \\
& +\left(\frac{241}{1260}+\frac{73 \sqrt{2}}{2520}-\frac{73 \sqrt{3}}{3780}-\frac{8 \sqrt{5}}{45}-\frac{73 \sqrt{6}}{7560}\right) \mu_{\Xi^{0}}+\left(\frac{1}{15}+\frac{\sqrt{2}}{30}-\frac{\sqrt{3}}{45}-\frac{\sqrt{6}}{90}\right) \mu_{\Delta^{++}}, \\
& \frac{1}{\sqrt{15}} \mu_{\Lambda \Sigma^{* 0}}^{(s)}=-\frac{\sqrt{15}}{45} \mu_{p}-\frac{\sqrt{15}}{27} \mu_{n}-\frac{7 \sqrt{15}}{135} \mu_{\Sigma^{+}}-\frac{\sqrt{15}}{135} \mu_{\Sigma^{-}}-\frac{8 \sqrt{15}}{135} \mu_{\Xi^{0}} .
\end{aligned}
$$

Similarly, the sum rules of the $u$ - and $d$-flavor components of the magnetic moments and transition magnetic moments are given by (A2) in the Appendix. Here one notes that the flavor components of the transition magnetic moments $\mu_{p \Delta^{+}}$and $\mu_{n \Delta^{0}}$ satisfy the identities

$$
\mu_{p \Delta^{+}}^{(q)}=\mu_{n \Delta^{0}}^{(q)}, \quad(q=u, d, s),
$$

to yield $\mu_{p \Delta^{+}}=\mu_{n \Delta^{0}}$ as in (2.10). The identities (3.5) are consistent with the previous ones in Refs. [14,16]. We list the predictions for the $u-, d$-, and $s$-flavor components of the decuplet and octet magnetic moments in Table I, and those for the decuplet-to-octet transition magnetic moments in Table II, by using the sum rules (3.4) and (A2).

Next, the form factors of the decuplet baryons, with internal structure, are defined by the matrix elements of the EM currents

$$
\begin{aligned}
\left\langle p+q\left|J^{\mu}\right| p\right\rangle= & \bar{u}(p+q)\left(\gamma^{\mu} F_{1 B}\left(q^{2}\right)\right. \\
& \left.+\frac{i}{2 m_{B}} \sigma^{\mu \nu} q^{\nu} F_{2 B}\left(q^{2}\right)\right) u(p),
\end{aligned}
$$

where $u(p)$ is the spinor of the baryons and $q$ is the momentum transfer. Using the $s$-flavor charge operator in the EM currents as before, in the limit of zero momentum transfer, one can obtain the strange form factors of baryon decuplet and octet

$$
F_{1 B}^{(s)}(0)=S, \quad F_{2 B}^{(s)}(0)=-3 \mu_{B}^{(s)}-S
$$

in terms of the strangeness quantum number of the baryon $S(=1-Y)(Y$, hypercharge $)$ and the strange components of the baryon decuplet and octet magnetic moments $\mu_{B}^{(s)}$. The predictions for the strange form factors of the decuplet 
and octet baryons are listed in Table I by using the relation (3.7).

\section{CONCLUSIONS}

In summary, we have derived sum rules for the baryon decuplet and octet magnetic moments and the decuplet-tooctet transition magnetic moments, in the chiral models with the SU(3) flavor group. These sum rules are explicitly constructed in terms of the six experimentally known baryon magnetic moments $\mu_{p}, \mu_{n}, \mu_{\Sigma^{+}}, \mu_{\Sigma^{-}}, \mu_{\Xi^{0}}$, and $\mu_{\Delta^{++}}$to yield the theoretical predictions for the remnant baryon magnetic moments. Especially in the case of using the experimental data for the six baryon magnetic moments as input data of the sum rules, the predicted value of $\mu_{\Omega^{-}}$is comparable to its experimental datum.

Next, we have extended the above algorithm to flavor components and strange form factors of the baryon decuplet and octet magnetic moments to find their sum rules in terms of the six baryon magnetic moments. The sum rules for the decuplet-to-octet transition magnetic moments and their flavor components have also been obtained. It is also shown that all the baryon decuplet and octet magnetic moments fulfill the model independent relations in the $u$ and $d$-flavor components and the $I$-spin symmetry of the isomultiplets with the same strangeness in the $s$-flavor channel. Moreover, the $s$-flavor components of the decuplet-to-octet transition magnetic moments respect the $I$-spin symmetry of the isomultiplets with the same strangeness. However, some transition magnetic moments such as $\mu_{p \Delta^{+}}^{(u)}, \mu_{n \Delta^{0}}^{(u)}$, and $\mu_{\Lambda \Sigma^{* 0}}^{(u)}$ do not satisfy the model independent relations in the $u$ - and $d$-flavor components. It is also interesting to see that $\mu_{p \Delta^{+}}^{(q)}$ and $\mu_{n \Delta^{0}}^{(q)}$ are equal to each other in the $q$-flavor $(q=u, d, s)$ channels, which are consistent with the previous results $[14,16]$.

It would be desirable if the $\mathrm{SU}(3)$ representation mixing effects on the baryon magnetic moments [24] could be investigated by exploiting the multiquark structure associated with the highly nontrivial nonlinear symmetry breaking terms.

\section{ACKNOWLEDGMENTS}

The author would like to deeply thank R. D. McKeown for the warm hospitality at Kellogg Radiation Laboratory, Caltech where a part of this work has been done. He is also grateful to G. E. Brown, H. C. Kim, R. D. McKeown, D. P. Min, B. Y. Park, M. Ramsey-Musolf, M. Rho, and P. Wang for helpful discussions and encouragements. This work was supported by the Korea Research Foundation Grant funded by the Korean Government (MOEHRD, Basic Research Promotion Fund), Grant No. KRF-2006-331C00071, and by the Korea Research Council of Fundamental Science and Technology (KRCF), Grant No. C-RESEARCH-2006-11-NIMS.

\section{APPENDIX}

In the $u$ - and $d$-flavor channels of the adjoint representation, the baryon magnetic moments and transition magnetic moments are given by

$$
\begin{aligned}
& \mu_{\Delta^{++}}^{(u)}=-2 \mu_{\Delta^{-}}^{(d)}=\frac{5}{12} \mathcal{M}+\frac{1}{3}\left(\mathcal{N}-\frac{1}{2 \sqrt{3}} \mathcal{N}^{\prime}\right)+\frac{2}{7} \mathcal{P}-\frac{1}{28} \mathcal{Q}-\frac{1}{21} \mathcal{R}, \\
& \mu_{\Delta^{+}}^{(u)}=-2 \mu_{\Delta^{0}}^{(d)}=\frac{3}{8} \mathcal{M}+\frac{1}{6}\left(\mathcal{N}-\frac{1}{2 \sqrt{3}} \mathcal{N}^{\prime}\right)+\frac{10}{63} \mathcal{P}+\frac{1}{126} \mathcal{Q}-\frac{1}{126} \mathcal{R}, \\
& \mu_{\Delta^{0}}^{(u)}=-2 \mu_{\Delta^{+}}^{(d)}=\frac{1}{3} \mathcal{M}+\frac{2}{63} \mathcal{P}+\frac{13}{252} \mathcal{Q}+\frac{2}{63} \mathcal{R} \\
& \mu_{\Delta^{-}}^{(u)}=-2 \mu_{\Delta^{++}}^{(d)}=\frac{7}{24} \mathcal{M}-\frac{1}{6}\left(\mathcal{N}-\frac{1}{2 \sqrt{3}} \mathcal{N}^{\prime}\right)-\frac{2}{21} \mathcal{P}+\frac{2}{21} \mathcal{Q}+\frac{1}{14} \mathcal{R}, \\
& \mu_{\Sigma^{*+}}^{(u)}=-2 \mu_{\Sigma^{*-}}^{(d)}=\frac{3}{8} \mathcal{M}+\frac{1}{6}\left(\mathcal{N}-\frac{1}{2 \sqrt{3}} \mathcal{N}^{\prime}\right)+\frac{19}{126} \mathcal{P}-\frac{17}{252} \mathcal{Q}-\frac{1}{63} \mathcal{R}, \\
& \mu_{\Sigma^{* 0}}^{(u)}=-2 \mu_{\Sigma^{* 0}}^{(d)}=\frac{1}{3} \mathcal{M}+\frac{1}{126} \mathcal{P}-\frac{1}{126} \mathcal{Q}+\frac{1}{126} \mathcal{R}, \\
& \mu_{\Sigma^{*-}}^{(u)}=-2 \mu_{\Sigma^{*+}}^{(d)}=\frac{7}{24} \mathcal{M}-\frac{1}{6}\left(\mathcal{N}-\frac{1}{2 \sqrt{3}} \mathcal{N}^{\prime}\right)-\frac{17}{126} \mathcal{P}+\frac{13}{252} \mathcal{Q}+\frac{2}{63} \mathcal{R}, \\
& \mu_{\Xi^{* 0}}^{(u)}=-2 \mu_{\Xi^{*-}}^{(d)}=\frac{1}{3} \mathcal{M}-\frac{1}{63} \mathcal{P}-\frac{17}{252} \mathcal{Q}-\frac{1}{63} \mathcal{R}, \\
& \mu_{\Xi^{*-}}^{(u)}=-2 \mu_{\Xi^{* 0}}^{(d)}=\frac{7}{24} \mathcal{M}-\frac{1}{6}\left(\mathcal{N}-\frac{1}{2 \sqrt{3}} \mathcal{N}^{\prime}\right)-\frac{11}{63} \mathcal{P}+\frac{1}{126} \mathcal{Q}-\frac{1}{126} \mathcal{R}, \\
& \mu_{\Omega^{-}}^{(u)}=-2 \mu_{\Omega^{-}}^{(d)}=\frac{7}{24} \mathcal{M}-\frac{1}{6}\left(\mathcal{N}-\frac{1}{2 \sqrt{3}} \mathcal{N}^{\prime}\right)-\frac{3}{14} \mathcal{P}-\frac{1}{28} \mathcal{Q}-\frac{1}{21} \mathcal{R},
\end{aligned}
$$




$$
\begin{aligned}
& \mu_{p}^{(u)}=-2 \mu_{n}^{(d)}=\frac{2}{5} \mathcal{M}+\frac{8}{45}\left(\mathcal{N}+\frac{1}{2} \mathcal{N}^{\prime}\right)+\frac{16}{135} \mathcal{P}-\frac{4}{135} \mathcal{Q}-\frac{2}{135} \mathcal{R} \\
& \mu_{n}^{(u)}=-2 \mu_{p}^{(d)}=\frac{11}{30} \mathcal{M}-\frac{2}{15}\left(\mathcal{N}+\frac{1}{2} \mathcal{N}^{\prime}\right)-\frac{2}{27} \mathcal{P}+\frac{7}{135} \mathcal{Q}+\frac{2}{135} \mathcal{R}, \\
& \mu_{\Sigma^{+}}^{(u)}=-2 \mu_{\Sigma^{-}}^{(d)}=\frac{2}{5} \mathcal{M}+\frac{8}{45}\left(\mathcal{N}+\frac{1}{2} \mathcal{N}^{\prime}\right)+\frac{26}{135} \mathcal{P}-\frac{2}{135} \mathcal{Q}-\frac{4}{135} \mathcal{R} \text {, } \\
& \mu_{\Sigma^{0}}^{(u)}=-2 \mu_{\Sigma^{0}}^{(d)}=\frac{19}{60} \mathcal{M}+\frac{1}{15}\left(\mathcal{N}+\frac{1}{2} \mathcal{N}^{\prime}\right)+\frac{11}{135} \mathcal{P}+\frac{1}{54} \mathcal{Q}+\frac{2}{135} \mathcal{R}, \\
& \mu_{\Sigma^{-}}^{(u)}=-2 \mu_{\Sigma^{+}}^{(d)}=\frac{7}{30} \mathcal{M}-\frac{2}{45}\left(\mathcal{N}+\frac{1}{2} \mathcal{N}^{\prime}\right)-\frac{4}{135} \mathcal{P}+\frac{7}{135} \mathcal{Q}+\frac{8}{135} \mathcal{R}, \\
& \mu_{\Xi^{0}}^{(u)}=-2 \mu_{\Xi^{-}}^{(d)}=\frac{11}{30} \mathcal{M}-\frac{2}{15}\left(\mathcal{N}+\frac{1}{2} \mathcal{N}^{\prime}\right)-\frac{22}{135} \mathcal{P}-\frac{2}{135} \mathcal{Q}+\frac{2}{135} \mathcal{R}, \\
& \mu_{\Xi^{-}}^{(u)}=-2 \mu_{\Xi^{0}}^{(d)}=\frac{7}{30} \mathcal{M}-\frac{2}{45}\left(\mathcal{N}+\frac{1}{2} \mathcal{N}^{\prime}\right)-\frac{8}{135} \mathcal{P}-\frac{4}{135} \mathcal{Q}-\frac{8}{135} \mathcal{R} \text {, } \\
& \mu_{\Lambda}^{(u)}=-2 \mu_{\Lambda}^{(d)}=\frac{7}{20} \mathcal{M}-\frac{1}{15}\left(\mathcal{N}+\frac{1}{2} \mathcal{N}^{\prime}\right)-\frac{1}{15} \mathcal{P}-\frac{1}{30} \mathcal{Q} \\
& \frac{1}{\sqrt{5}} \mu_{p \Delta^{+}}^{(u)}=\frac{1}{\sqrt{5}} \mu_{n \Delta^{0}}^{(u)}=\frac{1}{3 \sqrt{5}} \mathcal{M}-\frac{4}{45}\left(\mathcal{N}+\frac{2-\sqrt{2}}{8} \mathcal{N}^{\prime}\right)-\frac{8}{135} \mathcal{P}+\frac{7}{270} \mathcal{Q}+\frac{79}{4050} \mathcal{R}, \\
& \frac{1}{\sqrt{5}} \mu_{\Sigma^{+} \Sigma^{*+}}^{(u)}=-\frac{2}{\sqrt{5}} \mu_{\Sigma^{-} \Sigma^{*-}}^{(d)}=\frac{1}{3 \sqrt{5}} \mathcal{M}+\frac{4}{45}\left(\mathcal{N}+\frac{2-\sqrt{2}}{8} \mathcal{N}^{\prime}\right)+\frac{13}{135} \mathcal{P}+\frac{1}{270} \mathcal{Q}-\frac{1}{150} \mathcal{R}, \\
& \frac{1}{\sqrt{5}} \mu_{\Sigma^{0} \Sigma^{* 0}}^{(u)}=-\frac{2}{\sqrt{5}} \mu_{\Sigma^{0} \Sigma^{* 0}}^{(d)}=\frac{1}{3 \sqrt{5}} \mathcal{M}+\frac{2}{45}\left(\mathcal{N}+\frac{2-\sqrt{2}}{8} \mathcal{N}^{\prime}\right)+\frac{7}{135} \mathcal{P}+\frac{2}{135} \mathcal{Q}+\frac{1}{135} \mathcal{R}, \\
& \frac{1}{\sqrt{5}} \mu_{\Sigma^{-} \Sigma^{*-}}^{(u)}=-\frac{2}{\sqrt{5}} \mu_{\Sigma^{+} \Sigma^{*+}}^{(d)}=\frac{1}{3 \sqrt{5}} \mathcal{M}+\frac{1}{135} \mathcal{P}+\frac{7}{270} \mathcal{Q}+\frac{29}{1350} \mathcal{R}, \\
& \frac{1}{\sqrt{5}} \mu_{\Xi^{0} \Xi^{* 0}}^{(u)}=-\frac{2}{\sqrt{5}} \mu_{\Xi^{-} \Xi^{*-}}^{(d)}=\frac{1}{3 \sqrt{5}} \mathcal{M}+\frac{4}{45}\left(\mathcal{N}+\frac{2-\sqrt{2}}{8} \mathcal{N}^{\prime}\right)+\frac{14}{135} \mathcal{P}-\frac{1}{270} \mathcal{Q}+\frac{23}{4050} \mathcal{R}, \\
& \frac{1}{\sqrt{5}} \mu_{\Xi^{-} \Xi^{*-}}^{(u)}=-\frac{2}{\sqrt{5}} \mu_{\Xi^{0} \Xi^{* 0}}^{(d)}=\frac{1}{3 \sqrt{5}} \mathcal{M}+\frac{1}{135} \mathcal{P}+\frac{7}{270} \mathcal{Q}+\frac{67}{4050} \mathcal{R}, \\
& \frac{1}{\sqrt{15}} \mu_{\Lambda \Sigma^{* 0}}^{(u)}=\frac{1}{3 \sqrt{15}} \mathcal{M}-\frac{2}{45}\left(\mathcal{N}+\frac{2-\sqrt{2}}{8} \mathcal{N}^{\prime}\right)-\frac{1}{27} \mathcal{P}+\frac{2}{135} \mathcal{Q}+\frac{11}{2025} \mathcal{R}, \\
& \frac{1}{\sqrt{5}} \mu_{p \Delta^{+}}^{(d)}=\frac{1}{\sqrt{5}} \mu_{n \Delta^{0}}^{(d)}=-\frac{1}{6 \sqrt{5}} \mathcal{M}-\frac{2}{45}\left(\mathcal{N}+\frac{2-\sqrt{2}}{8} \mathcal{N}^{\prime}\right)-\frac{4}{135} \mathcal{P}+\frac{7}{540} \mathcal{Q}+\frac{79}{8100} \mathcal{R}, \\
& \frac{1}{\sqrt{15}} \mu_{\Lambda \Sigma^{* 0}}^{(d)}=-\frac{1}{6 \sqrt{15}} \mathcal{M}-\frac{1}{45}\left(\mathcal{N}+\frac{2-\sqrt{2}}{8} \mathcal{N}^{\prime}\right)-\frac{1}{54} \mathcal{P}+\frac{1}{135} \mathcal{Q}+\frac{11}{4050} \mathcal{R}
\end{aligned}
$$

Next, we list the sum rules of the $u$ - and $d$-flavor components of the magnetic moments and transition magnetic moments 


$$
\begin{aligned}
& \mu_{\Delta^{++}}^{(u)}=\frac{2}{3} \mu_{p}+\frac{10}{9} \mu_{n}+\frac{14}{9} \mu_{\Sigma^{+}}+\frac{2}{9} \mu_{\Sigma^{-}}+\frac{16}{9} \mu_{\Xi^{0}}+\frac{2}{3} \mu_{\Delta^{++}}, \\
& \mu_{\Delta^{+}}^{(u)}=\frac{11}{14} \mu_{p}+\frac{335}{252} \mu_{n}+\frac{103}{63} \mu_{\Sigma^{+}}+\frac{53}{126} \mu_{\Sigma^{-}}+\frac{443}{252} \mu_{\Xi^{0}}+\frac{1}{3} \mu_{\Delta^{++}} \text {, } \\
& \mu_{\Delta^{0}}^{(u)}=\frac{19}{21} \mu_{p}+\frac{65}{42} \mu_{n}+\frac{12}{7} \mu_{\Sigma^{+}}+\frac{13}{21} \mu_{\Sigma^{-}}+\frac{73}{42} \mu_{\Xi^{0}}, \\
& \mu_{\Delta^{-}}^{(u)}=\frac{43}{42} \mu_{p}+\frac{445}{252} \mu_{n}+\frac{113}{63} \mu_{\Sigma^{+}}+\frac{103}{126} \mu_{\Sigma^{-}}+\frac{433}{252} \mu_{\Xi^{0}}-\frac{1}{3} \mu_{\Delta^{++}}, \\
& \mu_{\Sigma^{*+}}^{(u)}=-\frac{11}{21} \mu_{p}+\frac{5}{28} \mu_{n}+\frac{137}{42} \mu_{\Sigma^{+}}+\frac{31}{42} \mu_{\Sigma^{-}}+\frac{271}{84} \mu_{\Xi^{0}}+\frac{1}{3} \mu_{\Delta^{++}} \text {, } \\
& \mu_{\Sigma^{* 0}}^{(u)}=\frac{13}{42} \mu_{p}+\frac{55}{63} \mu_{n}+\frac{271}{126} \mu_{\Sigma^{+}}+\frac{29}{63} \mu_{\Sigma^{-}}+\frac{142}{63} \mu_{\Xi^{0}}, \\
& \mu_{\Sigma^{*-}}^{(u)}=\frac{8}{7} \mu_{p}+\frac{395}{252} \mu_{n}+\frac{131}{126} \mu_{\Sigma^{+}}+\frac{23}{126} \mu_{\Sigma^{-}}+\frac{323}{252} \mu_{\Xi^{0}}-\frac{1}{3} \mu_{\Delta^{++}}, \\
& \mu_{\Xi^{* 0}}^{(u)}=-\frac{2}{7} \mu_{p}+\frac{25}{126} \mu_{n}+\frac{163}{63} \mu_{\Sigma^{+}}+\frac{19}{63} \mu_{\Sigma^{-}}+\frac{349}{126} \mu_{\Xi^{0}}, \\
& \mu_{\Xi^{*-}}^{(u)}=\frac{53}{42} \mu_{p}+\frac{115}{84} \mu_{n}+\frac{2}{7} \mu_{\Sigma^{+}}-\frac{19}{42} \mu_{\Sigma^{-}}+\frac{71}{84} \mu_{\Xi^{0}}-\frac{1}{3} \mu_{\Delta^{++}}, \\
& \mu_{\Omega^{-}}^{(u)}=\frac{29}{21} \mu_{p}+\frac{295}{252} \mu_{n}-\frac{59}{126} \mu_{\Sigma^{+}}-\frac{137}{126} \mu_{\Sigma^{-}}+\frac{103}{252} \mu_{\Xi^{0}}-\frac{1}{3} \mu_{\Delta^{++}} \text {, } \\
& \mu_{p}^{(u)}=\frac{4}{3} \mu_{p}+\frac{10}{9} \mu_{n}+\frac{14}{9} \mu_{\Sigma^{+}}+\frac{2}{9} \mu_{\Sigma^{-}}+\frac{16}{9} \mu_{\Xi^{0}}, \\
& \mu_{n}^{(u)}=\frac{2}{3} \mu_{p}+\frac{16}{9} \mu_{n}+\frac{14}{9} \mu_{\Sigma^{+}}+\frac{2}{9} \mu_{\Sigma^{-}}+\frac{16}{9} \mu_{\Xi^{0}}, \\
& \mu_{\Sigma^{+}}^{(u)}=\frac{2}{3} \mu_{p}+\frac{10}{9} \mu_{n}+\frac{20}{9} \mu_{\Sigma^{+}}+\frac{2}{9} \mu_{\Sigma^{-}}+\frac{16}{9} \mu_{\Xi^{0}}, \\
& \mu_{\Sigma^{0}}^{(u)}=\frac{2}{3} \mu_{p}+\frac{10}{9} \mu_{n}+\frac{17}{9} \mu_{\Sigma^{+}}+\frac{5}{9} \mu_{\Sigma^{-}}+\frac{16}{9} \mu_{\Xi^{0}}, \\
& \mu_{\Sigma^{-}}^{(u)}=\frac{2}{3} \mu_{p}+\frac{10}{9} \mu_{n}+\frac{14}{9} \mu_{\Sigma^{+}}+\frac{8}{9} \mu_{\Sigma^{-}}+\frac{16}{9} \mu_{\Xi^{0}}, \\
& \mu_{\Xi^{0}}^{(u)}=\frac{2}{3} \mu_{p}+\frac{10}{9} \mu_{n}+\frac{14}{9} \mu_{\Sigma^{+}}+\frac{2}{9} \mu_{\Sigma^{-}}+\frac{22}{9} \mu_{\Xi^{0}}, \\
& \mu_{\Xi^{-}}^{(u)}=\frac{2}{3} \mu_{p}+\frac{8}{9} \mu_{n}-\frac{2}{9} \mu_{\Sigma^{+}}-\frac{8}{9} \mu_{\Sigma^{-}}+\frac{2}{9} \mu_{\Xi^{0}}, \\
& \mu_{\Lambda}^{(u)}=\frac{2}{3} \mu_{n}+\frac{7}{3} \mu_{\Sigma^{+}}+\frac{1}{3} \mu_{\Sigma^{-}}+\frac{8}{3} \mu_{\Xi^{0}}, \\
& \frac{1}{\sqrt{5}} \mu_{p \Delta^{+}}^{(u)}=\frac{1}{\sqrt{5}} \mu_{n \Delta^{0}}^{(u)} \\
& =\left(-\frac{2}{567}+\frac{17 \sqrt{2}}{210}-\frac{17 \sqrt{3}}{315}+\frac{2 \sqrt{5}}{15}-\frac{17 \sqrt{6}}{630}\right) \mu_{p}+\left(\frac{346}{1701}+\frac{\sqrt{2}}{252}-\frac{\sqrt{3}}{378}+\frac{2 \sqrt{5}}{9}-\frac{\sqrt{6}}{756}\right) \mu_{n} \\
& +\left(\frac{3383}{17010}-\frac{\sqrt{2}}{630}+\frac{\sqrt{3}}{945}+\frac{14 \sqrt{5}}{45}+\frac{\sqrt{6}}{1890}\right) \mu_{\Sigma^{+}}+\left(\frac{2189}{17010}-\frac{13 \sqrt{2}}{630}+\frac{13 \sqrt{3}}{945}+\frac{2 \sqrt{5}}{45}+\frac{13 \sqrt{6}}{1890}\right) \mu_{\Sigma^{-}} \\
& +\left(\frac{2131}{17010}-\frac{73 \sqrt{2}}{1260}+\frac{73 \sqrt{3}}{1890}+\frac{16 \sqrt{5}}{45}+\frac{73 \sqrt{6}}{3780}\right) \mu_{\Xi^{0}}+\left(-\frac{2}{15}-\frac{\sqrt{2}}{15}+\frac{2 \sqrt{3}}{45}+\frac{\sqrt{6}}{45}\right) \mu_{\Delta^{++}},
\end{aligned}
$$




$$
\begin{aligned}
& \frac{1}{\sqrt{5}} \mu_{\Sigma^{+} \Sigma^{*+}}^{(u)}=\left(-\frac{83}{945}-\frac{17 \sqrt{2}}{210}+\frac{17 \sqrt{3}}{315}+\frac{2 \sqrt{5}}{15}+\frac{17 \sqrt{6}}{630}\right) \mu_{p}+\left(-\frac{1}{567}-\frac{\sqrt{2}}{252}+\frac{\sqrt{3}}{378}+\frac{2 \sqrt{5}}{9}+\frac{\sqrt{6}}{756}\right) \mu_{n} \\
& +\left(\frac{131}{1134}+\frac{\sqrt{2}}{630}-\frac{\sqrt{3}}{945}+\frac{14 \sqrt{5}}{45}-\frac{\sqrt{6}}{1890}\right) \mu_{\Sigma^{+}}+\left(\frac{107}{1134}+\frac{13 \sqrt{2}}{630}-\frac{13 \sqrt{3}}{945}+\frac{2 \sqrt{5}}{45}-\frac{13 \sqrt{6}}{1890}\right) \mu_{\Sigma^{-}} \\
& +\left(-\frac{589}{5670}+\frac{73 \sqrt{2}}{1260}-\frac{73 \sqrt{3}}{1890}+\frac{16 \sqrt{5}}{45}-\frac{73 \sqrt{6}}{3780}\right) \mu_{\Xi^{0}}+\left(\frac{2}{15}+\frac{\sqrt{2}}{15}-\frac{2 \sqrt{3}}{45}-\frac{\sqrt{6}}{45}\right) \mu_{\Delta^{++}}, \\
& \frac{1}{\sqrt{5}} \mu_{\Sigma^{0} \Sigma^{* 0}}^{(u)}=\left(\frac{11}{210}-\frac{17 \sqrt{2}}{420}+\frac{17 \sqrt{3}}{630}+\frac{2 \sqrt{5}}{15}+\frac{17 \sqrt{6}}{1260}\right) \mu_{p}+\left(\frac{3}{28}-\frac{\sqrt{2}}{504}+\frac{\sqrt{3}}{756}+\frac{2 \sqrt{5}}{9}+\frac{\sqrt{6}}{1512}\right) \mu_{n} \\
& +\left(\frac{19}{210}+\frac{\sqrt{2}}{1260}-\frac{\sqrt{3}}{1890}+\frac{14 \sqrt{5}}{45}-\frac{\sqrt{6}}{3780}\right) \mu_{\Sigma^{+}}+\left(\frac{37}{210}+\frac{13 \sqrt{2}}{1260}-\frac{13 \sqrt{3}}{1890}+\frac{2 \sqrt{5}}{45}-\frac{13 \sqrt{6}}{3780}\right) \mu_{\Sigma^{-}} \\
& +\left(-\frac{13}{420}+\frac{73 \sqrt{2}}{2520}-\frac{73 \sqrt{3}}{3780}+\frac{16 \sqrt{5}}{45}-\frac{73 \sqrt{6}}{7560}\right) \mu_{\Xi^{0}}+\left(\frac{1}{15}+\frac{\sqrt{2}}{30}-\frac{\sqrt{3}}{45}-\frac{\sqrt{6}}{90}\right) \mu_{\Delta^{++}}, \\
& \frac{1}{\sqrt{5}} \mu_{\Sigma^{-} \Sigma^{*-}}^{(u)}=\left(\frac{26}{135}+\frac{2 \sqrt{5}}{15}\right) \mu_{p}+\left(\frac{35}{162}+\frac{2 \sqrt{5}}{9}\right) \mu_{n}+\left(\frac{53}{810}+\frac{14 \sqrt{5}}{45}\right) \mu_{\Sigma^{+}}+\left(\frac{209}{810}+\frac{2 \sqrt{5}}{45}\right) \mu_{\Sigma^{-}}+\left(\frac{17}{405}+\frac{16 \sqrt{5}}{45}\right) \mu_{\Xi^{0}} \text {, } \\
& \frac{1}{\sqrt{5}} \mu_{\Xi^{0} \Xi^{* 0}}^{(u)}=\left(-\frac{1334}{2835}-\frac{17 \sqrt{2}}{210}+\frac{17 \sqrt{3}}{315}+\frac{2 \sqrt{5}}{15}+\frac{17 \sqrt{6}}{630}\right) \mu_{p}+\left(-\frac{451}{1701}-\frac{\sqrt{2}}{252}+\frac{\sqrt{3}}{378}+\frac{2 \sqrt{5}}{9}+\frac{\sqrt{6}}{756}\right) \mu_{n} \\
& +\left(\frac{2759}{3402}+\frac{\sqrt{2}}{630}-\frac{\sqrt{3}}{945}+\frac{14 \sqrt{5}}{45}-\frac{\sqrt{6}}{1890}\right) \mu_{\Sigma^{+}}+\left(\frac{1385}{3402}+\frac{13 \sqrt{2}}{630}-\frac{13 \sqrt{3}}{945}+\frac{2 \sqrt{5}}{45}-\frac{13 \sqrt{6}}{1890}\right) \mu_{\Sigma^{-}} \\
& +\left(\frac{8033}{17010}+\frac{73 \sqrt{2}}{1260}-\frac{73 \sqrt{3}}{1890}+\frac{16 \sqrt{5}}{45}-\frac{73 \sqrt{6}}{3780}\right) \mu_{\Xi^{0}}+\left(\frac{2}{15}+\frac{\sqrt{2}}{15}-\frac{2 \sqrt{3}}{45}-\frac{\sqrt{6}}{45}\right) \mu_{\Delta^{++}}, \\
& \frac{1}{\sqrt{5}} \mu_{\Xi^{-} \Xi^{*-}}^{(u)}=\left(\frac{98}{405}+\frac{2 \sqrt{5}}{15}\right) \mu_{p}+\left(\frac{125}{486}+\frac{2 \sqrt{5}}{9}\right) \mu_{n}+\left(-\frac{181}{2430}+\frac{14 \sqrt{5}}{45}\right) \mu_{\Sigma^{+}}+\left(\frac{407}{2430}+\frac{2 \sqrt{5}}{45}\right) \mu_{\Sigma^{-}} \\
& +\left(-\frac{109}{1215}+\frac{16 \sqrt{5}}{45}\right) \mu_{\Xi^{0}}, \\
& \frac{1}{\sqrt{15}} \mu_{\Lambda \Sigma^{* 0}}^{(u)}=\left(\frac{215}{1134}+\frac{17 \sqrt{2}}{420}-\frac{17 \sqrt{3}}{630}-\frac{17 \sqrt{6}}{1260}+\frac{2 \sqrt{15}}{45}\right) \mu_{p}+\left(\frac{1399}{6804}+\frac{\sqrt{2}}{504}-\frac{\sqrt{3}}{756}-\frac{\sqrt{6}}{1512}+\frac{2 \sqrt{15}}{27}\right) \mu_{n} \\
& +\left(-\frac{3751}{17010}-\frac{\sqrt{2}}{1260}+\frac{\sqrt{3}}{1890}+\frac{\sqrt{6}}{3780}+\frac{14 \sqrt{15}}{135}\right) \mu_{\Sigma^{+}}+\left(-\frac{1093}{17010}-\frac{13 \sqrt{2}}{1260}+\frac{13 \sqrt{3}}{1890}+\frac{13 \sqrt{6}}{3780}+\frac{2 \sqrt{15}}{135}\right) \mu_{\Sigma^{-}} \\
& +\left(-\frac{5779}{34020}-\frac{73 \sqrt{2}}{2520}+\frac{73 \sqrt{3}}{3780}+\frac{73 \sqrt{6}}{7560}+\frac{16 \sqrt{15}}{135}\right) \mu_{\Xi^{0}}+\left(-\frac{1}{15}-\frac{\sqrt{2}}{30}+\frac{\sqrt{3}}{45}+\frac{\sqrt{6}}{90}\right) \mu_{\Delta^{++}}, \\
& \frac{1}{\sqrt{5}} \mu_{p \Delta^{+}}^{(d)}=\frac{1}{\sqrt{5}} \mu_{n \Delta^{0}}^{(d)} \\
& =\left(-\frac{1}{567}+\frac{17 \sqrt{2}}{420}-\frac{17 \sqrt{3}}{630}-\frac{\sqrt{5}}{15}-\frac{17 \sqrt{6}}{1260}\right) \mu_{p}+\left(\frac{173}{1701}+\frac{\sqrt{2}}{504}-\frac{\sqrt{3}}{756}-\frac{\sqrt{5}}{9}-\frac{\sqrt{6}}{1512}\right) \mu_{n} \\
& +\left(\frac{3383}{34020}-\frac{\sqrt{2}}{1260}+\frac{\sqrt{3}}{1890}-\frac{7 \sqrt{5}}{45}+\frac{\sqrt{6}}{3780}\right) \mu_{\Sigma^{+}}+\left(\frac{2189}{34020}-\frac{13 \sqrt{2}}{1260}+\frac{13 \sqrt{3}}{1890}-\frac{\sqrt{5}}{45}+\frac{13 \sqrt{6}}{3780}\right) \mu_{\Sigma}- \\
& +\left(\frac{2131}{34020}-\frac{73 \sqrt{2}}{2520}+\frac{73 \sqrt{3}}{3780}-\frac{8 \sqrt{5}}{45}+\frac{73 \sqrt{6}}{7560}\right) \mu_{\Xi^{0}}+\left(-\frac{1}{15}-\frac{\sqrt{2}}{30}+\frac{\sqrt{3}}{45}+\frac{\sqrt{6}}{90}\right) \mu_{\Delta^{++}},
\end{aligned}
$$




$$
\begin{aligned}
\frac{1}{\sqrt{15}} \mu_{\Lambda \Sigma^{* 0}}^{(d)}= & \left(\frac{215}{2268}+\frac{17 \sqrt{2}}{840}-\frac{17 \sqrt{3}}{1260}-\frac{17 \sqrt{6}}{2520}-\frac{\sqrt{15}}{45}\right) \mu_{p}+\left(\frac{1399}{13608}+\frac{\sqrt{2}}{1008}-\frac{\sqrt{3}}{1512}-\frac{\sqrt{6}}{3024}-\frac{\sqrt{15}}{27}\right) \mu_{n} \\
& +\left(-\frac{3751}{34020}-\frac{\sqrt{2}}{2520}+\frac{\sqrt{3}}{3780}+\frac{\sqrt{6}}{7560}-\frac{7 \sqrt{15}}{135}\right) \mu_{\Sigma^{+}}+\left(-\frac{1093}{34020}-\frac{13 \sqrt{2}}{2520}+\frac{13 \sqrt{3}}{3780}+\frac{13 \sqrt{6}}{7560}-\frac{\sqrt{15}}{135}\right) \mu_{\Sigma^{-}} \\
& +\left(-\frac{5779}{68040}-\frac{73 \sqrt{2}}{5040}+\frac{73 \sqrt{3}}{7560}+\frac{73 \sqrt{6}}{15120}-\frac{8 \sqrt{15}}{135}\right) \mu_{\Xi^{0}}+\left(-\frac{1}{30}-\frac{\sqrt{2}}{60}+\frac{\sqrt{3}}{90}+\frac{\sqrt{6}}{180}\right) \mu_{\Delta^{++}}
\end{aligned}
$$

[1] R. Frisch and O. Stern, Z. Phys. 85, 4 (1933).

[2] S. Coleman and S. L. Glashow, Phys. Rev. Lett. 6, 423 (1961).

[3] A. Bosshard et al., Phys. Rev. D 44, 1962 (1991).

[4] H. T. Diehl et al., Phys. Rev. Lett. 67, 804 (1991).

[5] D. B. Leinweber, T. Draper, and R. M. Woloshyn, Phys. Rev. D 46, 3067 (1992).

[6] F. Schlumpf, Phys. Rev. D 48, 4478 (1993); J. Linde and H. Snellman, Phys. Rev. D 53, 2337 (1996).

[7] S. T. Hong and G.E. Brown, Nucl. Phys. A580, 408 (1994).

[8] M. N. Butler, M. J. Savage, and R. P. Springer, Phys. Rev. D 49, 3459 (1994).

[9] F. X. Lee, Phys. Lett. B 419, 14 (1998); Phys. Rev. D 57, 1801 (1998).

[10] J. Linde, T. Ohlsson, and H. Snellman, Phys. Rev. D 57, 5916 (1998).

[11] G. S. Yang, H. C. Kim, M. Praszalowicz, and K. Goeke, Phys. Rev. D 70, 114002 (2004).

[12] E. Jenkins, M. Luke, A. V. Manohar, and M. Savage, Phys. Lett. B 302, 482 (1993).

[13] S. J. Puglia, M. J. Ramsey-Musolf, and S. L. Zhu, Phys. Rev. D 63, 034014 (2001).

[14] E. Jenkins and A. V. Manohar, Phys. Lett. B 335, 452 (1994).

[15] E. Jenkins, X. Ji, and A. V. Manohar, Phys. Rev. Lett. 89, 242001 (2002).

[16] R. F. Lebed and D. R. Martin, Phys. Rev. D 70, 016008 (2004).

[17] H. C. Kim, M. Polyakov, M. Praszalowicz, G. S. Yang, and K. Goeke, Phys. Rev. D 71, 094023 (2005).
[18] D. T. Spayde et al. (SAMPLE Collaboration), Phys. Lett. B 583, 79 (2004).

[19] R. D. McKeown, Phys. Lett. B 219, 140 (1989); E. J. Beise and R. D. McKeown, Comments Nucl. Part. Phys. 20, 105 (1991); R. D. McKeown, in New Directions in Quantum Chromodynamics, edited by C. R. Ji and D. P. Min (AIP, Melville, New York, 1999).

[20] A. Acha et al. (HAPPEX Collaboration), Phys. Rev. Lett. 98, 032301 (2007).

[21] R. D. McKeown, arXiv:hep-ph/9607340.

[22] G.E. Brown and M. Rho, Phys. Lett. B 82, 177 (1979).

[23] S. T. Hong and B. Y. Park, Nucl. Phys. A561, 525 (1993); S. T. Hong, B. Y. Park, and D. P. Min, Phys. Lett. B 414, 229 (1997).

[24] S. T. Hong and Y. J. Park, Phys. Rep. 358, 143 (2002), and references therein.

[25] R. F. Dashen, E. Jenkins, and A. V. Manohar, Phys. Rev. D 49, 4713 (1994); R. Dashen, E. Jenkins, and A. V. Manohar, Phys. Rev. D 51, 3697 (1995); J. Dai, R. Dashen, E. Jenkins, and A. V. Manohar, Phys. Rev. D 53, 273 (1996).

[26] G. 't Hooft, Nucl. Phys. B72, 461 (1974).

[27] E. Witten, Nucl. Phys. B160, 57 (1979).

[28] R. Flores-Mendieta, E. Jenkins, and A. V. Manohar, Phys. Rev. D 58, 094028 (1998).

[29] M. A. B. Beg, B. W. Lee, and A. Pais, Phys. Rev. Lett. 13, 514 (1964).

[30] S. T. Hong and G.E. Brown, Nucl. Phys. A564, 491 (1993). 